\title{
Preface from the Major Chairpersons
}

The 2014 Workshop on Advances in the Turin Shroud Investigation (ATSI 2014) took place in Bari (Italy) in date 4-5 September 2014 to address typical issues of this fascinating subject. The event was organized by the Department of Electrical and Information Engineering of the Technical University of Bari with the logistic support of the University of Bari which made a noble multifunctional Center available to the attendees. ATSI 2014 was originally aimed at offering an opportunity to present and then debate some novel research results concerned with image formation and processing, dating, textile's manufacturing and physicochemical aging, applied electrostatics and electromagnetism, to name a few. Topics of equal intellectual value such as, but not limited to, forensic medicine, archaeology, history, philosophy and iconography was then added to the list. Of the 30 contributions submitted to peer review, only 19 was accepted for presentation and discussion during the workshop. The Official Program for this two-day forum is reported in http://dee.poliba.it/ATSI2014/index.htm. Later, the Major Chairpersons made the unscheduled decision to select 9 of the above contributions in order for them to be re-examined with a view to a possible publication on SHS Web of Conferences. The reference Authors of each of the 9 selected papers were asked to revise their papers taking into account comments and questions raised both after presentations and during informal discussions through the workshop. Finally, the revised papers were submitted to a second round of peer-review and modified according to Reviewers' remarks.

This restricted collection of papers essentially cover a number of topics distinctive of the Turin Shroud (TS) studies, notably in-situ and laboratory investigation on TS-like electrostatic imaging; micro-scale optical observation and macro-scale reproduction; TS coloration, conservation and pattern perception; commonalities and coincidences with the Oviedo Sudarium; archaeological survey on funerary textiles in ancient Israel with a comparison with the TS; historiographical contribution to the debated question of the burial cloths reported in the Gospel account for the benefit of related applications, e.g. Liturgy and Iconography.

This SHS Web of Conferences issue has been successfully assembled taking advantage, on one hand, of the sympathetic willingness of the involved Authors and anonymous Reviewers, and on the other of the Workshop Official Sponsorship's invaluable additional support. To all these Colleagues and Institutions, the undersigned Chairs owe an unforgettable debt of gratitude.

\section{ATSI 2014 General Chairs:}

Bruno Barberis, University of Turin, Italy and also Director of International Center of the Turin Shroud Studies (CIS), Turin, Italy;

Francesco Lattarulo, Technical University of Bari, Italy

ATSI 2014 Program Chairs:

Paolo Di Lazzaro, ENEA, Frascati Research Center, Italy;

Dario Petri, University of Trento, Italy

This is an Open Access article distributed under the terms of the Creative Commons Attribution License 4.0, which permits unrestricted use, distribution, and reproduction in any medium, provided the original work is properly cited. 